\title{
Gas accretion from the cosmic web in the local Universe
}

\author{
J. Sánchez Almeida ${ }^{1,2}$, B. G. Elmegreen ${ }^{3}$, C. Muñoz-Tuñón ${ }^{1,2}$ and \\ D. M. Elmegreen ${ }^{4}$ \\ ${ }^{1}$ Instituto de Astrofísica de Canarias, E-38205 La Laguna, Tenerife, Spain \\ email: jos@iac.es, cmt@iac.es \\ ${ }^{2}$ Departamento de Astrofísica, Universidad de La Laguna, Tenerife, Spain \\ ${ }^{3}$ IBM Research Division, T.J. Watson Research Center, Yorktown Heights, NY 10598, USA \\ email: bge@us.ibm.com \\ ${ }^{4}$ Department of Physics and Astronomy, Vassar College, Poughkeepsie, NY 12604, USA \\ email: elmegreen@vassar.edu
}

\begin{abstract}
Numerical simulations predict that gas accretion from the cosmic web drives star formation in disks galaxies. The process is important in low mass haloes $\left(<10^{12} \mathrm{M}_{\odot}\right)$, therefore, in the early universe when galaxies were low mass, but also in dwarf galaxies of the local universe. The gas that falls in is predicted to be tenuous, patchy, partly ionized, multi-temperature, and large-scale; therefore, hard to show in a single observation. One of the most compelling cases for gas accretion at work in the local universe comes from the extremely metal poor (XMP) galaxies. They show metallicity inhomogeneities associated with star-forming regions, so that large starbursts have lower metallicity than the underlying galaxy. Here we put forward the case for gas accretion from the web posed by XMP galaxies. Two other observational results are discussed too, namely, the fact that the gas consumption time-scale is shorter than most stellar ages, and the systematic morphological distortions of the HI around galaxies.
\end{abstract}

Keywords. Galaxies: evolution and Galaxies: formation and Galaxies: general and Galaxies: high-redshift and Galaxies: star formation and large-scale structure of Universe

\section{Star-formation and gas accretion from the cosmic web}

Galaxies are not just test particles tracing the cosmic web. Galaxies consume cosmic gas by accretion, and also modify the chemical composition of the web through winds that spread out debris from stellar evolution. Numerical simulations predict that accretion of metal-poor gas from the cosmic web fuels the formation of disk galaxies (e.g., Dekel et al. 2009, Genel et al. 2012). This cosmological gas supply has a strong dependence on halo mass, and so, on redshift. When the gas encounters a massive halo $\left(>10^{12} \mathrm{M}_{\odot}\right)$, it becomes shock heated and requires a long time to cool and settle into the galaxy disk. For less massive haloes, cool gas streams can reach the inner halo or disk directly through the so-called cold-flow accretion. Since high redshift haloes tend to be low in mass, cold-flow accretion is predicted to be the main mode of galaxy growth in early times. Ultimately, the galaxies evolve into a quasi-stationary state, where inflows and outflows balance the star formation rate (SFR), a phase that still goes on for most of them.

Although the theoretical predictions are clear, we only have indirect evidence for gas infall feeding star-formation. These evidences are reviewed by Sánchez Almeida et al. (2014a), and we refer to this work for full details. Here we illustrate the type of evidence (Sect. 1), emphasizing our contribution to the issue, which is related to inhomogeneities in the distribution of metallicity found in local XMP galaxies (Sect. 2). Other recent reviews covering cosmic gas accretion from different perspectives have been written by 
Sancisi et al. (2008), Silk \& Mamon (2012), Combes (2014), Fraternali (2014), Conselice et al. (2013), Benson (2010), and Madau \& Dickinson (2014).

\section{Observational evidence for accretion in the local Universe}

Two examples are discussed, chosen to be representative of a long list of indirect evidence put forward by Sánchez Almeida et al. (2014a).

Gas consumption time-scale shorter than stellar ages. The Kennicutt-Schmidt (KS) law (Kennicutt 1998) provides a time-scale to consume the gas available to form stars $\tau_{g}$. According to the KS law, the SFR scales as a power of the gas mass $\mathrm{M}_{\mathrm{g}}$, with power index close to one, i.e.,

$$
\mathrm{SFR} \simeq \frac{\mathrm{M}_{\mathrm{g}}}{\tau_{g}} .
$$

If a galaxy evolves as a closed box, $\tau_{g}$ gives the time-scale to exhaust the gas available to form stars; $\tau_{g}$ is measured to change from 0.5 to 2 Gyr for galaxies in the redshift range between 2 and 0 (e.g., Genzel et al. 2010; Gnedin et al. 2014). When outflows are important, as expected in dwarf galaxies (e.g., Peeples \& Shankar 2011), the gas consumption time-scale can be much shorter than $\tau_{g}$ (e.g., Sánchez Almeida et al. 2014a, Sect. 2.1). Despite this very short gas-consumption time-scale, star-forming galaxies are observed to have stars of all ages from the origin of the Universe to the present (e.g., Heavens et al. 2004; Sánchez Almeida et al. 2012). The only way to reconcile this short gas-consumption time-scale with the ages of the stars is assuming a continuous gas infall able to replace the gas lost through star-formation and winds.

Morphological distortions of the HI distribution. Neutral gas is present around almost all galaxies. Often the HI maps present non-axisymmetric distortions showing that the gas is not contained in a disk or a spheroid and so, suggesting the gas distribution to be transient. Spirals are known to have extended warped HI envelopes that can be sustained by gas infall (e.g., Bournaud et al. 2005). Extreme distortions are actually common among galaxies with large specific SFR in the local universe (Lelli et al. 2012; Nidever et al. 2013), with kinematically separate components, streamers extending far beyond the optical size, kinematic HI axes offset from the optical axes, and clouds associated with recent starbursts (Johnson et al. 2012; Ashley et al. 2013). A particularly telling deviation from axi-symmetry has been found recently by Kacprzak et al. (2012). They report a bimodality in the azimuthal angle distribution of low ionization gas around galaxies as traced by MgII absorption along QSO lines-of-sight. The circum-galactic gas prefers to lie near the projected galaxy major and minor axes. The bimodality is clear in blue star-forming galaxies whereas red passive galaxies exhibit an excess of absorption along their major axis. These results suggest the bimodality to be driven by gas accretion along the galaxy major axis and gas outflows along the minor axis, with no other clear alternative explanation existing yet.

\section{Evidence from extremely metal poor galaxies}

The secular evolution of disk galaxies produces a regular pattern with the metallicity decreasing inside out, i.e., having a negative gradient with galactocentric distance (Vilchez et al. 1988). The time-scale for gas mixing in a disk is fairly short, on the order of a rotational period or a few hundred Myr. Deviations from negative metallicity gradients are attributed to the recent arrival of cosmic gas that feeds the star formation. If the gas is accreted through the cold-flow mode, it is expected to reach the disks in clumps 

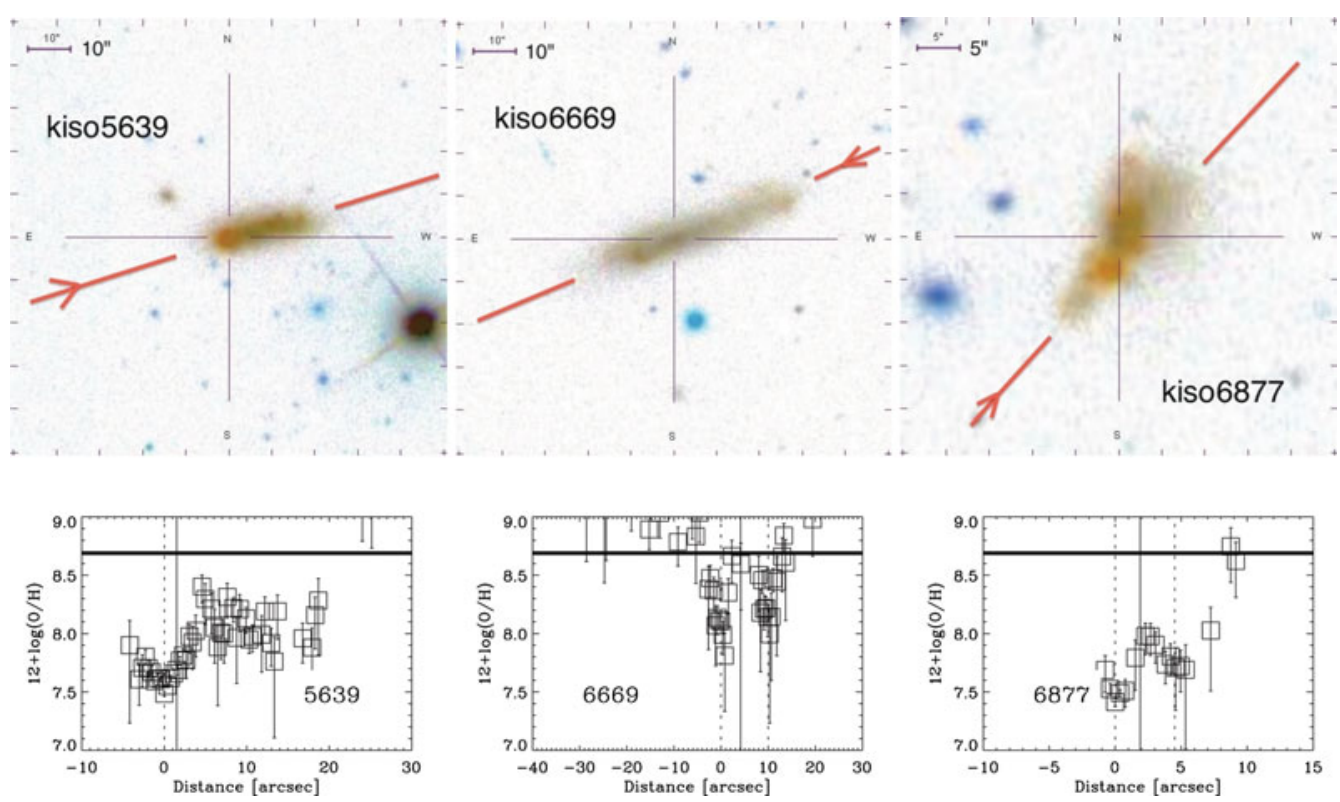

Figure 1. Top: Images of three tadpole galaxies characterized by having a bright peripheral clump on a faint tail. The images have been taken from SDSS, and are displayed with an inverted color palette so that the background sky looks white, and the intrinsically blue galaxies appear reddish. The red line shows the orientation of the spectrograph slit used to measure metallicity variations, and the horizontal scales on the upper left corner of the panels correspond to 5 or 10 arcsec as indicated. Bottom: Oxygen abundance variation across the galaxies on top. The vertical solid line represents the center of rotation, whereas the vertical dotted lines indicate the location of maxima in SFR. Note the existence of abundance variations, with the minima coinciding with the regions of largest SFRs. The thick horizontal solid line indicates the solar metallicity.

often forming stars already (e.g., Dekel et al. 2009; Ceverino et al. 2010; Genel et al. 2012). Alternatively, the external gas streams may fuel the disks with metal-poor gas, so that gas mass builds up developing starbursts through internal gravitational instabilities (e.g., Noguchi 1999; Bournaud \& Elmegreen 2009). In any case, the cold-flow accretion is bound to induce metal-poor starbursts.

Metallicity drops associated with intense starbursts may reflect cold-flow accretion. Cresci et al. (2010) were the first to identify this pattern in high redshift galaxies. The same kind of metallicity drops appear in local tadpole galaxies, which are often XMPs (Morales-Luis et al. 2011). The bright star-forming head of the XMP tadpole has lower metallicity than the underlying galaxy (see Fig. 1). Sánchez Almeida et al. (2013) interpret this observation as an episode of gas accretion onto the tadpole head. Localized metallicity drops associated with star-forming regions have also been observed in other objects, including gamma ray burst host galaxies (Levesque et al. 2011; Thöne et al. 2014), BCD galaxies (Izotov et al. 2009; Sánchez Almeida et al. 2014b) and dIrr galaxies (Haurberg et al. 2013). Variations of metallicity among HII regions located at the same galactrocentric distance are not unusual even in large nearby spirals (e.g., Li et al. 2013); some of these variations could come from localized accretion events. 


\section{Take-home message}

Numerical simulations tell us that most star-formation in spirals is driven by gas accretion from the cosmic web. It is a process happening at all redshifts. Although this is a solid theoretical prediction, only indirect evidence exist so far. The case of the XMP galaxies results particularly compelling. XMP galaxies seems to be primitive disks in the process of assembling in the nearby universe, where a major cold-flow accretion episode is producing the current starburst. They are extreme cases showing a physical process that affects many other disk galaxies.

\section{References}

Ashley, T., Simpson, C. E., \& Elmegreen, B. G. 2013, AJ, 146, 42

Benson, A. J. 2010, Physics Reports, 495, 33

Bournaud, F., Combes, F., Jog, C. J., \& Puerari, I. 2005, A\&\&A, 438, 507

Bournaud, F. \& Elmegreen, B. G. 2009, ApJ, 694, L158

Ceverino, D., Dekel, A., \& Bournaud, F. 2010, MNRAS, 404, 2151

Combes, F. 2014, in Astronomical Society of the Pacific Conference Series, Vol. 480, Structure and Dynamics of Disk Galaxies, ed. M. S. Seigar \& P. Treuthardt, 211

Conselice, C. J., Mortlock, A., Bluck, A. F. L., Grützbauch, R., \& Duncan, K. 2013, MNRAS, 430,1051

Cresci, G., Mannucci, F., Maiolino, R., et al. 2010, Nat, 467, 811

Dekel, A., Birnboim, Y., Engel, G., et al. 2009, Nat, 457, 451

Fraternali, F. 2014, in IAU Symposium, Vol. 298, IAU Symposium, ed. S. Feltzing, G. Zhao, N. A. Walton, \& P. Whitelock, 228-239

Genel, S., Naab, T., Genzel, R., et al. 2012, ApJ, 745, 11

Genzel, R., Tacconi, L. J., Gracia-Carpio, J., et al. 2010, MNRAS, 407, 2091

Gnedin, N. Y., Tasker, E. J., \& Fujimoto, Y. 2014, ApJ, 787, L7

Haurberg, N. C., Rosenberg, J., \& Salzer, J. J. 2013, ApJ, 765, 66

Heavens, A., Panter, B., Jimenez, R., \& Dunlop, J. 2004, Nat, 428, 625

Izotov, Y. I., Guseva, N. G., Fricke, K. J., \& Papaderos, P. 2009, A\& A, 503, 61

Johnson, M., Hunter, D. A., Oh, S.-H., et al. 2012, AJ, 144, 152

Kacprzak, G. G., Churchill, C. W., \& Nielsen, N. M. 2012, ApJ, 760, L7

Kennicutt, Jr., R. C. 1998, ApJ, 498, 541

Lelli, F., Verheijen, M., Fraternali, F., \& Sancisi, R. 2012, A\&A, 544, A145

Levesque, E. M., Berger, E., Soderberg, A. M., \& Chornock, R. 2011, ApJ, 739, 23

Li, Y., Bresolin, F., \& Kennicutt, Jr., R. C. 2013, ApJ, 766, 17

Madau, P. \& Dickinson, M. 2014, ARA\&A, 52, 415

Morales-Luis, A. B., Sánchez Almeida, J., Aguerri, J. A. L., \& Muñoz-Tuñón, C. 2011, ApJ, 743,77

Nidever, D. L., Ashley, T., Slater, C. T., et al. 2013, ApJ, 779, L15

Noguchi, M. 1999, ApJ, 514, 77

Peeples, M. S. \& Shankar, F. 2011, MNRAS, 417, 2962

Sánchez Almeida, J., Elmegreen, B. G., Muñoz-Tuñón, C., \& Elmegreen, D. M. 2014a, A\&ARev, 22,71

Sánchez Almeida, J., Morales-Luis, A. B., Muñoz-Tuñón, C., et al. 2014b, ApJ, 783, 45

Sánchez Almeida, J., Muñoz-Tuñón, C., Elmegreen, D. M., Elmegreen, B. G., \& Méndez-Abreu, J. 2013, ApJ, 767, 74

Sánchez Almeida, J., Terlevich, R., Terlevich, E., Cid Fernandes, R., \& Morales-Luis, A. B. 2012, ApJ, 756, 163

Sancisi, R., Fraternali, F., Oosterloo, T., \& van der Hulst, T. 2008, AESARev, 15, 189

Silk, J. \& Mamon, G. A. 2012, Research in Astronomy and Astrophysics, 12, 917

Thöne, C. C., Christensen, L., Prochaska, J. X., et al. 2014, MNRAS, 441, 2034

Vilchez, J. M., Pagel, B. E. J., Diaz, A. I., Terlevich, E., \& Edmunds, M. G. 1988, MNRAS, 235,633 